\title{
Evaluation of Modified Turbo Spin Echo Technique Compared with Double Inversion Recovery Technique in Acquisition of Black Blood Brain Vessel Image
}

\author{
Kwan-Woo Choi ${ }^{1}$, Ho-Beom Lee ${ }^{1}$, Sa-Ra Na ${ }^{1}$, and Soon-Yong Son ${ }^{2 *}$ \\ ${ }^{1}$ Asan Medical Center, 88, Olympic-ro 43-gil, Songpa-gu, Seoul, Korea \\ ${ }^{2}$ Wonkwang Univ, Sinyong-dong, Iksan-si, Jeollabuk-do, Korea
}

(Received 27 October 2015, Received in final form 20 February 2016, Accepted 22 February 2016)

\begin{abstract}
The main goal was to evaluate effectiveness of a modified TSE sequence compared with DIR (double inversion recovery) sequence in acquisition of fast flow brain vessel images using signal void effect. 32 healthy volunteers (10 men and 22 women; mean age of 31 years; ranging between 28-43 years) who underwent black blood DIR sequence (group A) and the modified TSE sequence (group B) were enrolled in our study. Signal to Noise Ratio (SNR) and Contrast to Noise Ratio (CNR) of the internal carotid arteries' lumen were compared in T1 and T2 weighted images for both group A and B. The images obtained from group B showed lower SNR values in internal carotid artery than the group $A$ in both of the $\mathrm{T} 1$ and $\mathrm{T} 2$ weighted images $(11.49 \%$ and $13.66 \%$ respectively). While the CNR values were higher in the group B than the group $A$ in both of the T1 and T2 weighted images $(8.69 \%$ and $7.55 \%$ respectively). The qualitative score of all categories were not significantly different between the two groups. Furthermore approximately $49 \%$ of the total scan time was reduced from group B. Our study is to shorten the scanning time and minimize the inconveniences of the patients in acquisition of the black blood images of brain by using the signal void effect in the modified TSE technique while keeping the diagnostic value of the test.
\end{abstract}

Keywords : MRI, Black blood, flow void effect

\section{Introduction}

Shape of intracranial artery diseases is important to choose the treatment method due to its complexity. Typically, MRI techniques for the evaluation of vessel wall in the reported studies include black blood imaging [1-5]. Black blood techniques in which the signal from flowing blood is suppressed and the signal from the stationary tissues is retained provide an excellent contrast between vessel walls [6-14]. Black blood imaging is commonly performed using double inversion recovery (DIR) magnetization preparation $[15,16]$.

DIR and spatial saturation blood suppression rely on the inflow of blood with nulled signal into the imaging volume and are therefore a function of volume thickness, blood velocity and flow pattern.

However, it is limited by its long scan time due to the requirement of single-slice sequential acquisition. Also,

CThe Korean Magnetics Society. All rights reserved.

*Corresponding author: Tel: +82-2-3010-4325

Fax: +82-2-3010-6788, e-mail: son6392@hanmail.net the blood suppression is often insufficient in multi-slice studies. Previous studies have reported several methods used to acquire sufficient blood suppression within reasonable scan time. For example, several DIR preparation techniques have been recently developed, including multislice acquisition techniques. Also, parallel acquisition techniques reduce scan time by combining the signals of several coil elements in a phased array to reconstruct the image [17-22]. However, DIR still needs longer time and may lead to motion artifact which make radiologist misunderstand.

To our knowledge, The absence of signal for flowing spins is most pronounced only when the slices are oriented perpendicular to the direction of flow, and the flow velocities are high enough to be transported out of the slice in the duration between the $90^{\circ}$ and $180^{\circ}$ pulses. Therefore, simple SE sequences do not provide adequate suppression of blood signal in oblique orientations. However, Turbo spin echo (TSE) techniques provide excellent blood suppression for two-dimensional (2D) imaging of brain arteries with fast blood flow such as the carotid artery [23]. The purpose of our study was to compare the 
blood suppression between DIR black blood and modified TSE sequence of the internal carotid artery in volunteers.

\section{Materials and Methods}

\subsection{Study principle}

The ability of MR to image the cerebral vasculature continues to evolve and improve. Arteries are routinely visible on spin-echo images due to the "flow void" phenomenon. In order to collect a signal from flowing fluids, the protons must remain within the imaging volume for both the initial $90^{\circ}$ and the following $180^{\circ}$ refocusing radiofrequency (rf) pulses. In the case of rapid arterial flow, the protons leave the imaging volume between the two rf pulses, no signal is collected, and the arteries are rendered dark on the MR image. The flow-related signal loss is more likely to occur with pulse sequences using long echo times. On T2-weighted images the dark vessels are contrasted nicely with the high signal CSF within the cisterns and the intermediate signal of the brain parenchymal.

The "flow void" phenomenon is very helpful in evaluating cerebral vascular disease. Since normally the arteries are dark, the presence of any intraluminal signal suggests either a high-grade stenosis or thrombosis. Also, arteriovenous malformations (AVM) are readily detected on noncontrast MR scans because of the high flow through these lesions. Dilated vascular structures often identify the arterial supply and the venous drainage of the nidus of the malformation. Giant aneurysms $(\geq 2.5 \mathrm{~cm})$ are clearly imaged on MR. Smaller aneurysms are more of a challenge; thin sections and multiplanar imaging increases the yield (Fig .1) [24].

\subsection{Volunteers study}

From August 2012 to January 2013, 32 healthy volunteers $(10$ men and 22 women; mean age of 31 years; ranging between 28-43 years) underwent black blood imaging with the DIR sequence and the modified TSE sequence. Signal to Noise Ratio (SNR) and Contrast to Noise Ratio (CNR) of the internal carotid arteries lumen were compared in $\mathrm{T} 1$ and $\mathrm{T} 2$ weighted images to determine whether there are differences between the two techniques for depiction of the signal void effect inside the vessel wall.

\subsection{Image acquisition}

MRI scanning was performed on a 3.0T MRI scanner (Archieva, Philips Healthcare). The body coil was used as the transmitter, and a dedicated, eight-channel, phasedarray coil was used as the receiver. Axial-plane images

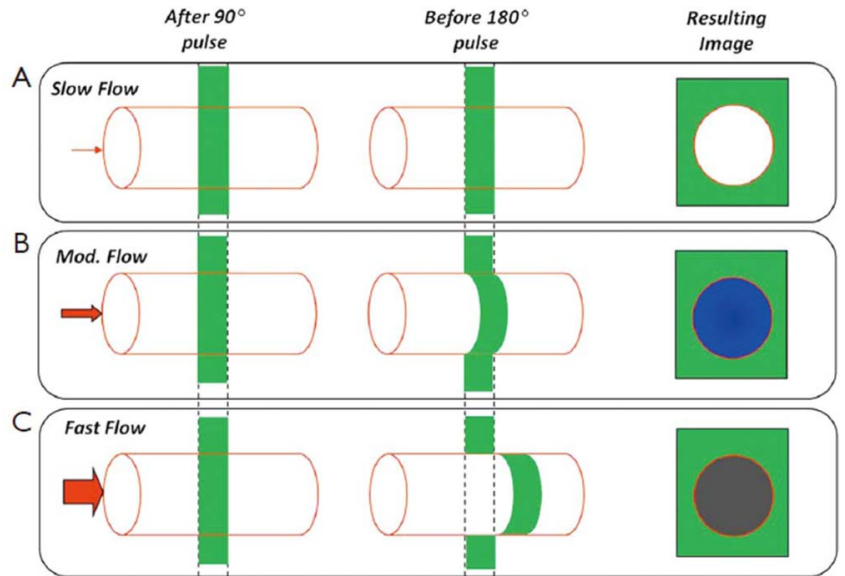

Fig. 1. (Color online) Spin echo based black-blood imaging based on flow void phenomenon. Through plane flow through a slice at different flow rates, slowest on the top panel and the fastest in the bottom panel. Slow flowing spins experience both the $90^{\circ}$ and the $180^{\circ}$ pulses, and therefore fully refocus to give a bright signal. On the other hand, if the spin velocity is high enough, none of the spins experience both the $90^{\circ}$ and the $180^{\circ}$ pulses resulting in a signal void within the vessel. If the spin velocity is in-between these two extremes, then the resulting image has a signal intensity that is proportional to the fraction of the spins that experienced both the $90^{\circ}$ and the $180^{\circ} \mathrm{RF}$ pulses. As a result slowly flowing spins near the vessel wall can refocus and make the vessel wall appear thicker, if the TE is too short.

were obtained along the long axis of internal carotid artery. Each method was peripheral-pulse gated, and was acquired with the following parameters.: modified T1 sequence (TR/TE, 1000/8.4; $1 \mathrm{~mm}$ thickness with a no gap; FOV, $100 \times 100$; matrix size, $200 \times 200$; number of slices. 10; number of excitations, 2; and flip angle, 90 degrees).

Modified T2 sequence (TR/TE, 2000/80; $1 \mathrm{~mm}$ thickness with a no gap; FOV, $100 \times 100$; matrix size, $200 \times$ 200; number of slices, 10; number of excitations, 2; and flip angle, 90 degrees). The scans were repeated using the same protocol: DIR black blood technique (group A) and modified TSE (group B) application of the black blood.

\subsection{Image Evaluation}

The SNR and CNR were measured in four ROIs with an area of $10 \mathrm{~mm}^{2}$. The ROIs were as follows: (1) ROI of the internal carotid artery; (2) ROI of the sphenoid sinus; (3) ROI of the white matter (Fig. 2). The SNR and CNR were calculated using the following equations: SNR = (lesion SI/background SD) $\times 100, \mathrm{CNR}=($ lesion SI-surrounding tissue SI)/background noise $\mathrm{SD} \times 100$.

Qualitative image analysis was performed by three radiologists, each with eight or more years of MRI clinical 


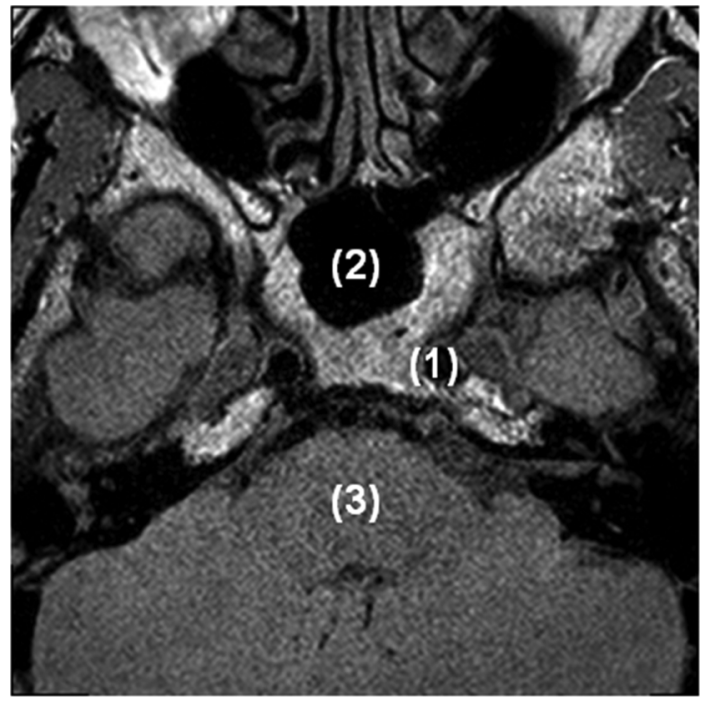

Fig. 2. 33-year-old male volunteer. A axial, black blood MRI image shows the position of the Regions of Interest (ROIs); (1) ROI of the internal carotid artery; (2) ROI of the sphenoid sinus; (3) ROI of the white matter.

experience, and who independently read the two sets of imaging with and without DIR pulse. All of the three radiologists were blinded to the volunteers' information. The images were evaluated for the overall quality of the atherosclerotic plaques as well as vessel wall and lumen. For each of the three categories listed, the qualitative image was graded on a scale of 1 to 4 as follows: grade 1 = excellent; grade 2 = more than adequate diagnosis; grade 3 = adequate; and grade $4=$ inadequate for diagnosis. The average rating from the three radiologists was calculated for the three categories.

\subsection{Statistical Analysis}

The statistical significance of the parametric data was determined using a paired sample $\mathrm{t}$ test. A two-sided $\mathrm{P}$ value less than 0.05 was considered to indicate statistical significance. The scores of the three image quality categories were analyzed using the Wilcoxon signed rank test. All statistical analysis was performed using the SPSS software package (version 18; SPSS, Chicago, IL, USA).

\section{Results}

The image obtained from group B showed lower SNR values of internal carotid artery than the group $\mathrm{A}$ in both of the T1 and T2 weighted images (11.49\% and $13.66 \%$ respectively). While the $\mathrm{CNR}$ values were higher in the group B than in the DIR images in both of the modified $\mathrm{T} 1$ and T2 weighted images $(8.69 \%$ and $7.55 \%$ respectively).
Table 1. Quantitative results of vessel wall images in two groups.

\begin{tabular}{ccccc}
\hline \multicolumn{2}{c}{ Category } & Group A & Group B & P value \\
\hline \multirow{2}{*}{ SNR } & T1 & $41.15 \pm 34.11$ & $36.42 \pm 29.92$ & .189 \\
& T2 & $44.15 \pm 39.57$ & $38.12 \pm 29.45$ & .061 \\
\hline \multirow{2}{*}{ CNR } & T1 & $173.17 \pm 148.48$ & $188.21 \pm 149.27$ & .234 \\
& T2 & $174.33 \pm 153.63$ & $161.16 \pm 116.70$ & .291 \\
\hline
\end{tabular}

Table 2. Qualitative results of vessel wall images in two groups.

\begin{tabular}{lccc}
\hline \hline & Group A & Group B & P value \\
\hline Blood suppression quality & $1.28 \pm 0.46$ & $1.41 \pm 0.50$ & .325 \\
Clarity of vessel wall and lumen & $1.41 \pm 0.61$ & $1.49 \pm 0.51$ & .701 \\
Overall image quality & $2.00 \pm 0.43$ & $1.93 \pm 0.25$ & .488 \\
\hline
\end{tabular}

Table 3. Scan time results of vessel wall image in two groups.

\begin{tabular}{ccc}
\hline \hline & & Average scan time \\
\hline \multirow{2}{*}{ Group A } & T1 & $6 \min 25 \mathrm{sec}$ \\
& T2 & $5 \min 50 \mathrm{sec}$ \\
\hline \multirow{2}{*}{ Group B } & T1 & $3 \min 35 \mathrm{sec}$ \\
& T2 & $2 \min 39 \mathrm{sec}$ \\
\hline
\end{tabular}

The quantitative results of the SNRs and CNRs of the two images are summarized in Table 1. There was no significant difference between the two groups for either of the SNR or CNR $(\mathrm{P}>0.01)$. The two groups were demonstrated having almost identical blood suppression patterns.

The results of the qualitative analysis are summarized in Table 2. The qualitative scores of all categories were not significantly different between the two groups. On the other hand, group A required longer scan time than group B. The results of the scan time are summarized in Table 3.

\section{Discussion}

In our study, the main goal was to evaluate the effectiveness of modified TSE sequence in depicting fast flow vessels. With use of 3.0Tesla MRI, we obtained sufficient blood suppression within a reasonable scan time. And we also confirmed that removal of mimicking blood artifacts using 2D MRI.

In previous studies, Song $\mathrm{HK}$ et al. investigated the efficiency of multi-slice DIR on black blood technique. They noted the multiple-slice DIR technique can reduce the scan time by a factor of two or more [11]. However the drawback of this study is reduction in SNR. Ryan Brown et al. [25] pointed out DIR blood suppression may be inadequate for vessel wall imaging in the vessel where 

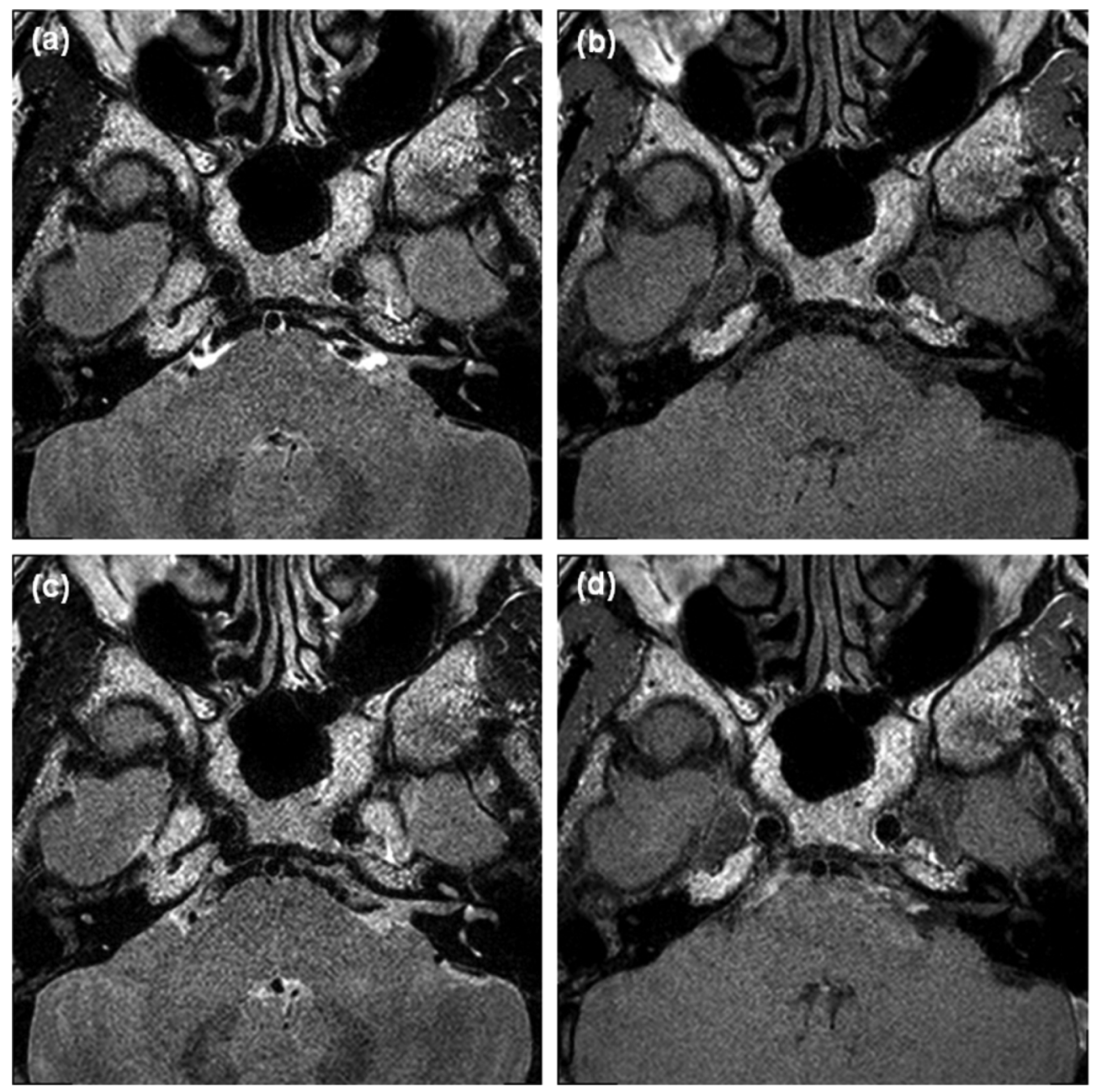

Fig. 3. 33-year-old male volunteer. axial, vessel wall MRI images show the blood suppression is maintained equal in DIR black blood imaging and modified TSE imaging on T1 and T2 weighted images, respectively. (a) Modified TSE T2-weighted image (b) Modified TSE T1-weighted image. (c) DIR black blood T2-weighted image. (d) DIR black blood T1-weighted image.

slow flowing blood's prominent. This inadequate blood suppression may result in wall-mimicking blood artifacts and inflated vessel wall area measurements.

The results of our study are clinically significant for the following reasons. First, the signals from fast flow vessels can be suppressed in group B sufficiently. Second, acceptable overall image quality can be acquired in most volunteers. Our study proved that novel black-blood images could be acquired in group B with SNR and CNR values similar to group A in internal carotid artery. Balu $\mathrm{N}$ et al. also indicated 2D high resolution vessel wall MR imaging can help assess the remodeling pattern and plaque distribution of vessel wall disease consequently [26].

Typical spin echo imaging utilizes only the initial echo for diagnosis. The initial echo contains signal from both stationary blood and vessel wall, which are difficult to differentiate. However, in rapid moving blood flow blood signals are voided. This is caused by a lack of refocusing of blood, which is excited by the $90^{\circ}$ pulse but not by the $180^{\circ}$ pulse $[11,23]$. In general definitions, high SNR and CNR are expected as a result of experiment. However, in our study the SNR meant the degree of the remaining blood signals as a result of incomplete blood saturation. The CNR meant the degree of the suppressed blood signal among lesions. There were no significant differences in both of the SNR and CNR between the TSE sequence and DIR black blood sequence.

Furthermore, another advantage of our study is that the group B (TSE sequence) requires shorter scan time than the DIR black blood sequence. The image obtained from group B showed shorter scan time in internal carotid artery than the group B in both of the T1 and T2 weighted images. Relatively longer scan time of group A (DIR sequences) often causes motion artifact. Motion leads to 
signal loss and inhomogeneity in images, which can affect diagnosis. Signal loss artifacts are thought to be due to misalignment of the black-blood preparation with readout TSE.

Our study has some limitations. Although patients suffering neurovascular disease often have reduced blood velocity due to stenosis or occlusion, we obtained only a limited number of healthy volunteers. Second, our evaluation was only performed on the cavernous segment of internal carotid artery; comparisons are needed in other segment of the internal carotid artery. We only covered 10 $\mathrm{mm}$ in our study (transverse slice) to compare the effectiveness of the two sequences. However in clinical environment larger coverage will be needed. Therefore in DIR sequence scan time will become even longer.

In spite of these limitations, the modified TSE is advantageous for fast imaging with vessel wall MRI and is useful for providing signal void effect. Thus it allowed high-quality image. In conclusion, the modified TSE technique using the flow void effect shortens the scanning time and minimizes the inconveniences of the patients in acquisition of the DIR black blood images while it keeps the diagnostic value of the test.

\section{Conclusion}

Signal to Noise Ratio (SNR) and Contrast to Noise Ratio (CNR) of the internal carotid arteries' lumen were compared in T1 and T2 weighted images for both group A and B. The images obtained from group B showed lower SNR values in internal carotid artery than the group A in both of the T1 and T2 weighted images (11.49\% and $13.66 \%$ respectively). While the CNR values were higher in the group B than the group A in both of the T1 and $\mathrm{T} 2$ weighted images $(8.69 \%$ and $7.55 \%$ respectively). The qualitative score of all categories were not significantly different between the two groups. Furthermore approximately $49 \%$ of the total scan time was reduced from group B. In conclusion, the modified TSE technique using the flow void effect shortens the scanning time and minimizes the inconveniences of the patients in acquisition of the DIR black blood images while it keeps the diagnostic value of the test.

\section{References}

[1] T. S. Hatsukami, R. Ross, N. L. Polissar, and C. Yuan, Circulation 102, 9 (2000).

[2] C. Yuan, J. W. Murakami, C. E. Hayes, J. S. Tsuruda, T. S. Hatsukami, K. S. Wildy, M. S. Ferguson, and D. E. Jr, Strandness, J. Magn. Reson. Imaging 5, 5 (1995).
[3] C. Yuan, J. S. Tsuruda, K. N. Beach, C. E. Hayes, M. S. Ferguson, C. E. Alpers, T. K. Foo, and D. E. Strandness, J. Magn. Reson. Imaging 4, 1 (1994).

[4] J. F. Toussaint, G. M. LaMuraglia, J. F. Southern, V. Fuster, and H. L. Kantor, Circulation 94, 5 (1996).

[5] W. B. Winn, U. P. Schmiedl, D. D. Reichenbach, K. W. Beach, H. Nghiem, C. Dimas, E. Daniel, K. R. Maravilla, and C. Yuan, AJNR Am. J. Neuroradiol 19, 1(1998).

[6] S. Sinha and U. Sinha, J. Magn. Reson. Imaging 6, 3 (1996).

[7] R. R. Edelman, D. Chien, and D. Kim, Radiology 181, 3 (1991).

[8] Y. Liu, S. J. Riederer, and R. L. Ehman, Magn. Reson. Med. 30, 2 (1993)

[9] S. Zhang, J. Cai, Y. Luo, C. Han, N. L. Polissar, T. S. Hatsukami, and C. Yuan, Radiology 228, 1 (2003).

[10] S. Zhang, T. S. Hatsukami, N. L. Polissar, C. Han, and C. Yuan, Radiology 19, 6 (2001).

[11] H. K. Song, A. C. Wright, R. L. Wolf, and F. W. Wehrli, Magn. Reson Med. 47, 3 (2002).

[12] D. L. Parker, K. C. Goodrich, M. Masiker, J. S. Tsuruda, and G. L. Katzman, Magn. Reson. Med. 47, 5 (2002).

[13] V. L. Yarnykh and C. Yuan, J. Magn. Reson. Imaging 17, 4 (2003).

[14] L. A. Crowe, P. Gatehouse, G. Z. Yang, R. H. Mohiaddin, A. Varghese, C. Charrier, J. Keegan, and D. N. Firmin, J. Magn. Reson. Imaging 17, 5 (2003).

[15] V. V. Itskovich, V. Mani, G. Mizsei, J. G. Aguinaldo, D. D. Samber, F. Macaluso, P. Wisdom, and Z. A. Fayad, J. Magn. Reson. Imaging 19, 4 (2004).

[16] J. P. Felmlee and R. L. Ehman, Radiology 164, 2 (1987).

[17] T. D. Nguyen, L. de Rochefort, P. Spincemaille, M. D. Cham, J. W. Weinsaft, M. R. Prince, and Y. Wang, J. Magn. Reson. Imaging 28, 5 (2008).

[18] I. Koktzoglou and D. Li, J. Cardiovas Magn. Reson. 9, 1 (2007).

[19] J. Wang, V. L. Yarnykh, T. Hatsukami, B. Chu, N. Balu, and C. Yuan, Magn. Reson. Med. 58, 5 (2007).

[20] J. H. Brittain, E. W. Olcott, A. Szuba, G. E. Gold, G. A. Wright, P. Irarrazaval, and D. G. Nishimura, Magn. Reson. Med. 38, 3 (1997).

[21] C. Y. Liu, O. Wieben, J. H. Brittain, and S. B. Reeder, J. Magn. Reson. Imaging 28, 5 (2008).

[22] C. Y. Liu, T. A. Bley, O. Wieben, J. H. Brittain, and S. B. Reeder, J. Magn. Reson. Imaging 31, 1 (2010).

[23] D. A. Steinman and B. K. Rutt, Magn. Reson. Med. 39, 4 (1998).

[24] R. Krishnamurthy, B. Cheong, and R. Muthupillai, Cardiovasc Diagn. Ther. 4, 2 (2014).

[25] R. Brown, T. D. Nguyen, P. Spincemaille, M. D. Cham, G. Choi, P. A. Winchester, M. R. Prince, and Y. Wang, Magn. Reson. Med. 63, 3 (2010).

[26] N. Balu, B. Chu, T. S. Hatsukami, C. Yuan, and V. L. Yarnykh, J. Magn. Reson. Imaging 27, 4 (2008). 